Author(s):

Submitted to:
Basil Swanson, CST-DO DeQuan Li, CST-4
RECEIVED

ОСТ 304996

OSTI

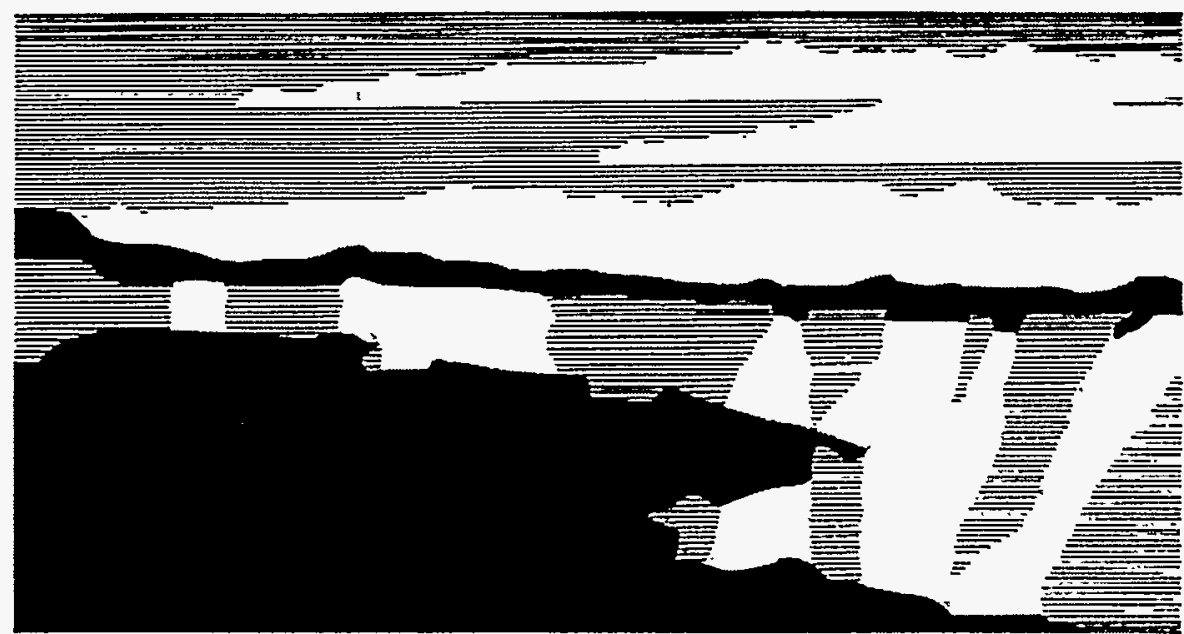

Los Alamos Natlonal Laboratory, an afflumattve action/equal opportunity employer, is operated by the University of Califomia for the U.S. Department of Energy under contract W-7405-ENG-36. By accoptance of this anticle, the publisher recognizes that the U.S. Govemmont retatns a nonexclustve, royaltyfree license to publish or reproduce the published form of this contrbutton, or to allow others to do so, for U.S. Govemment purposes. The Los Alamos Natbral Laboratory requests that the publsher identify this article as work pertormed under the ausplces of tho U.S. Department of Energy.

$$
\text { HH }
$$




\section{DISCLAIMER}

This report was prepared as an account of work sponsored by an agency of the United States Government. Neither the United States Government nor any agency thereof, nor any of their employees, makes any warranty, express or implied, or assumes any legal liability or responsibility for the accuracy, completeness, or usefulness of any information, apparatus, product, or process disclosed, or represents that its use would not infringe privately owned rights. Reference herein to any specific commercial product, process, or service by trade name, trademark, manufacturer, or otherwise does not necessarily constitute or imply its endorsement, recommendation, or favoring by the United States Government or any agency thereof. The views and opinions of authors expressed herein do not necessarily state or reflect those of the United States Government or any agency thereof. 


\title{
Self-Assembled Thin Film Chemical Sensors
}

\author{
Basil Swanson* and DeQuan Li
}

\begin{abstract}
This is the final report of a one-year, Laboratory-Directed Research and Development (LDRD) project at the Los Alamos National Laboratory (LANL). Current chemical sensors suffer from poor molecular specificity, sensitivity, and stability and seldom have the recovery properties needed for real-time monitoring applications. We have employed self-assembly techniques to covalently bond species-selective reagents directly to the surface of the transducer so that analyte/reagent chemistry occurs at the interface between the transducer and the media to be monitored. The use of self-assembling monolayer and -multilayer (SAM) techniques results in stable sensing elements with optimal specificity built in through the use of reagents that have been designed for molecular recognition. Moreover, self-assembly chemistry applied to oxide surfaces allows flexible means of transduction spanning optical, electrochemical, mass-loading, and conduction methods. The work conducted on this project focused on demonstration of the methodology and the application to selected organic vapors (aromatic compounds and halogenated hydrocarbons). We have been able to develop a series of surface acoustic wave (SAW) sensors that are specific for aromatic compounds and halogenated hydrocarbons based on self-assembled thin films of cyclodextrins and calixarenes. Monolayers of seven different cyclodextrins and clixarenes have been attached to SAW transducers and their response to several organic molecules in the vapor phase have been measured. This preliminary data confirms the efficacy of this approach for real-time monitoring of hydrocarbons.
\end{abstract}

\section{Background and Research Objectives}

Real-time monitoring of chemical species represents a stiff challenge for the sensor community. The desired attributes of chemical sensors for real-world applications include reversibility of analyte binding (for real-time sensing), specificity (for identification of the

\footnotetext{
* Principal investigator, e-mail: basil @lanl.gov
} 
species), high sensitivity, stability to hostile environments, and the ability to build sensor arrays for monitoring complex mixtures. The combination of sensitivity, reversibility, specificity, and stability is particularly difficult to achieve.

The focus of the sensor community for the past several years has been on transduction, instrumentation, intelligent signal processing for arrays, ruggidization for real-world applications, and cost reduction. As a result, very little attention has been paid to the critical piece of the sensor in terms of the above attributes--the sensor element itself. Most sensor elements used in current sensor technology have little specificity and many have poor sensitivity, stability, and reversibility. Only recently has the sensor community begun to appreciate the need for advanced materials and chemistry to design and fabricate better sensor elements. This project focused on design and fabrication of advanced sensor elements based on novel materials synthesis and molecular recognition.

Previous results from our laboratory established the groundwork for the fabrication of surface acoustic wave (SAW) chemical sensors for real-time monitoring of volatile organic compounds and, in particular, halogenated hydrocarbons. The approach was based on using self-assembly techniques to covalently bond species selective reagents directly to the surface of a SAW device. Given the need for reversibility as well as specificity and sensitivity, we focused on clyclodextrin molecules for the reagents. Cyclodextrins are naturally occurring species that are known to bind aromatic compounds and halogenated hydrocarbons to form 'host-guest' molecules. The binding of the organic by the cyclodextrin is reversible, thereby allowing real-time sensing. The selectivity of the cyclodextrins for particular organic compounds can be adjusted by the cavity size of the cyclodextrins $(\alpha, \beta, \gamma$ vary in size) and by functionalization of the rims of the cyclodextrin molecules. Our initial work was devoted to synthesis of selected cyclodextrins and the formation of monolayer thin films using selfassembly methods. These self-assembled monolayers (SAMs) were then tested against halogenated hydrocarbons and aromatic compounds that are known to be environmental pollutants. Although the monolayer thin films did not exhibit great sensitivity, they did allow us to map the specificity of the various cyclodextrin thin film SAW devices to a number of target organic materials.

The overall objectives of this LDRD project were to extend the monolayer SAMs to multilayer thin films to enhance sensitivity and to prepare additional molecule specific reagents based on calix(n)arenes. 


\section{Importance to LANL's Science and Technology Base and National R\&D Needs}

This project supports LANL core competencies in Earth and Environmental Systems and Nuclear and Advanced Materials. The need for advanced chemical sensors is ubiquitous and includes applications such as site characterization, post closure monitoring, process diagnostics and control, as well as stockpile maintenance and surveillance. This project represents a new paradigm for designing and fabricating smart chemical sensor elements. The general approach of self-assembly and molecular recognition can be extended not only to a number of other transduction methodologies (optical, electrochemical, conductance) but also to a number of different target chemical species (heavy metals, actinides, etc.). Given the breadth of chemical sensor needs, the Laboratory's capabilities in molecular recognition (e.g., ligand design) and materials synthesis (e.g., self-assembly and membrane synthesis) provide a unique opportunity to address critical needs of the DOE and the Nation.

\section{Scientific Approach and Results to Date}

We report here a novel approach to construct a new generation of superior chemical sensors based on tailoring the surface chemistry of the sensing layer on surface acoustic wave (SAW) devices using innovative molecular self-assembled monolayer (SAM) techniques. Such sensors are desired for cost-effective environmental monitoring, site remediation, and industrial process characterization. A versatile family of organic "bucket" molecules known as cyclodextrins were functionalized with a bifunctional organosilanes. The inside of the host cavity exhibits hydrophobic properties and, depending on the variety and functionalization, is just such a size and chemical environment to readily incorporate specific VOCs via host-guest interactions. 


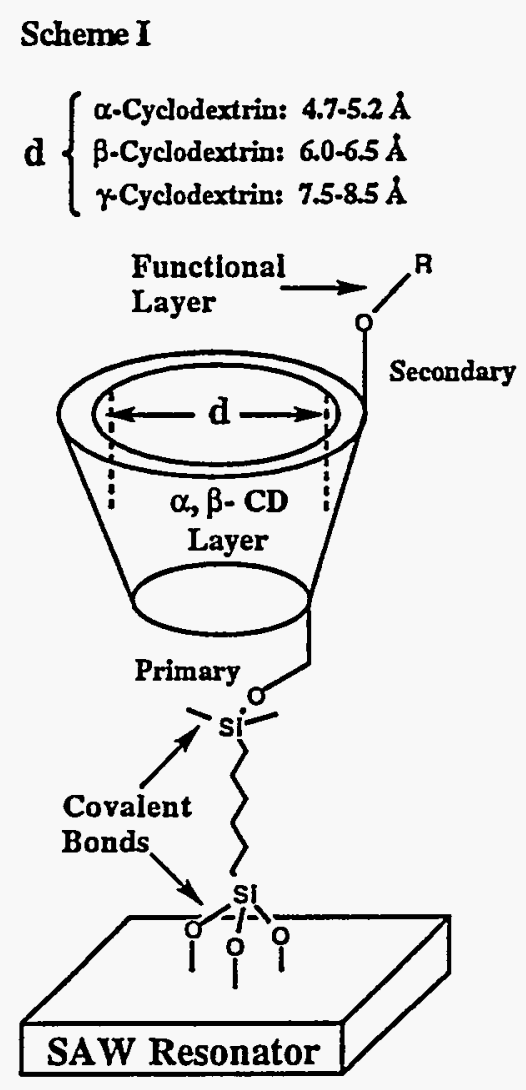

While previous works on SAW- and electrochemical-based applications of SAMs have relied on a thiol-gold linkage, we have chosen to employ a much stronger siloxane linkage that is generally appropriate for attachment to oxide surfaces terminated with hydroxyl groups. Using efficient molecular engineered cyclodextrin or calixarene host monolayers, we have achieved ultrahigh sensitivity comparable to that of thicker polymer spin-casted or spray-coated films. Current detection limit is about 20 parts per billion (ppb). The optimized interactions between hosts and guests are attributable to the proper alignment, lipophilic cavity, and functionalization of the molecular buckets. Numerous asymmetric host molecules are synthesized and used in the study of sensor application. A typical example is the functionalization of cyclodextrin with 3-isocyanatopropyltrimethoxylsilane and the is suitable for polymerization on surface in the form of sol-gel. The sol-gel film approach is based on the success of monolayer work, which includes $\alpha$-cyclodextrin dodeca(2-O,3-O)benzoate (1), $\beta$ cyclodextrin tetradeca $(2-O, 3-O)$ benzoate (2), $\beta$-cyclodextrin tetradeca(2-O,3-O)acetate (3), cundecylcalix[4]resorcinarene (4), and 37,38,39,40,41,42-hexahydroxycalix[6]arene (5). The host monolayer thin films were prepared by exposing the cleaned substrates to the vapor of 1,6-bis(trichlorosilyl)hexane by bubbling argon through a trap-bubbler-trap system at 150 $\mathrm{mL} / \mathrm{min}$ at $70^{\circ} \mathrm{C}$. The silane derivatized substrates were then immersed in the approximately 
1.0-5.0 mM corresponding cyclodextrin or calixarene solutions for about $2 \mathrm{hr}$ at room temperature to allow the formation of self-assembled host monolayers.

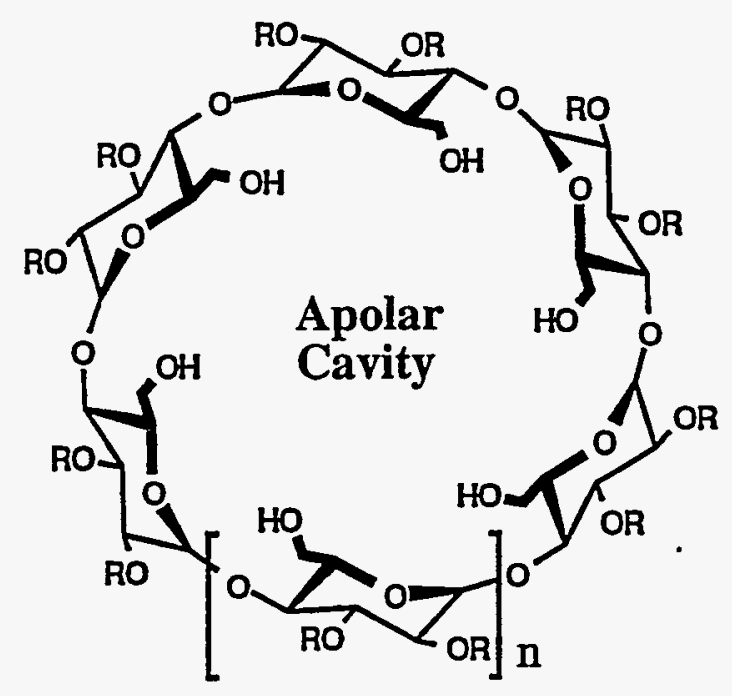

1: $\mathrm{R}=-\mathrm{COC}_{6} \mathrm{H}_{5}, \mathrm{n}=1$

2: $\mathrm{R}=-\mathrm{COC}_{6} \mathrm{H}_{5}, \mathrm{n}=2$

3: $\mathrm{R}=-\mathrm{COCH}_{3}, \mathrm{n}=2$<smiles>[2H]C([2H])c1cc([C@@H]([2H])c2cc([C@H]([2H])c3cc([C@H]([2H])c4cc([C@H]([2H])c5cc([C@H]([2H])c6cc(C([2H])[2H])c(O)cc6O)c(O)cc5O)c(O)cc4O)c(O)cc3O)c(O)cc2O)c(O)cc1O</smiles>

4: $\mathrm{R}^{\prime}=-\mathrm{CH}_{2}\left(\mathrm{CH}_{2}\right)_{9} \mathrm{CH}_{3}$<smiles>Oc1ccccc1Cc1cccc(Cc2ccccc2Cc2ccccc2Cc2cccc(Cc3ccccc3O)c2O)c1O</smiles>

5

Typically, two substrates were employed: Si wafers of $<100>$ orientation were used for surface FTIR studies and piezoelectric ST-cut quartz for the SAW studies to measure mass loading. The polarized, variable-angle, internal total-reflection infrared technique (PVAI-ATRIR) was used with an incidence of $45^{\circ}$ within a Ge hemisphere crystal in both p- and spolarized geometries. The coated surface can be readily verified by FTIR-ATR. For example, cyclodextrin-benzoate monolayer has the carbonyl and phenyl ring vibration region at 1738 , 1284 , and $708 \mathrm{~cm} 1$ (note that the $\mathrm{Si}$ wafer also absorbs at less than $1430 \mathrm{~cm}-1$ ), as well as features from the linker bis(trichlorosilyl)hexyl $\mathrm{CH}_{2}$ bands at 2926 , and $2855 \mathrm{~cm}-1$ in both pand s-polarized spectra. 
SAW resonators were used to measure both surface thin-film coverage and sensor response to analytes. The phase velocity of this acoustic wave-and therefore the resonant frequency of the device-is a sensitive function of the physical properties of any contacting materials. In the present case of thin, nonconductive monolayers having no lateral connectivity (i.e., covalent bonds), electrical and viscoelastic effects are minimal and the resonant frequency shift $(\Delta f)$ depends mainly on the mass loading per unit area $(\Delta \mathrm{m} / \mathrm{A})$ of the sensing monolayer and any adsorbed vapors according to

$$
\Delta \mathrm{f}=-\mathrm{K} \mathrm{f}^{2} /(\Delta \mathrm{m} / \mathrm{A})
$$

where $\mathrm{K}=1.3 \times 10^{-6} \mathrm{~s}^{\circ} \mathrm{cm}^{2} / \mathrm{g}$.

Figure 1 shows real-time reversible, rapid (on the order of seconds) sensor response to 1-minute-wide acetone pulses at the particular concentrations. Over five orders of vapor concentrations (approximately 300,000-1 ppm), a saturation behavior was observed at high organic vapor partial pressures; whereas sensor exhibits a linear response within a narrow concentration range, especially at low concentrations. Typical sensor responses to VOC partial pressure at $15.0 \pm 0.1^{\circ} \mathrm{C}$ gave frequency shifts much greater $8.0 \mathrm{kHz}$ for multilayer cyclodextrin coatings, corresponding to approximately a multilayer amounts of the VOC, suggesting an average of multiple analyte molecules per surface-attached cyclodextrin multilayer channel. As expected, the sensor responses (Df) increase almost linearly with the cyclodextrin thin-film thickness and ultrahigh sensitive VOC sensors were successfully demonstrated in this manner.

In conclusion, we have synthesized a number of asymmetric cyclodextrin derivatives and formed host thin films using silane coupling reagents. VOC sensors based on these cyclodextrin monolayers/multilayers were demonstrated to have middle-ppb sensitivity to targeted organic analytes. Pattern recognition with an array of microsensors appears to be a feasible approach for identifying a particular VOC. 


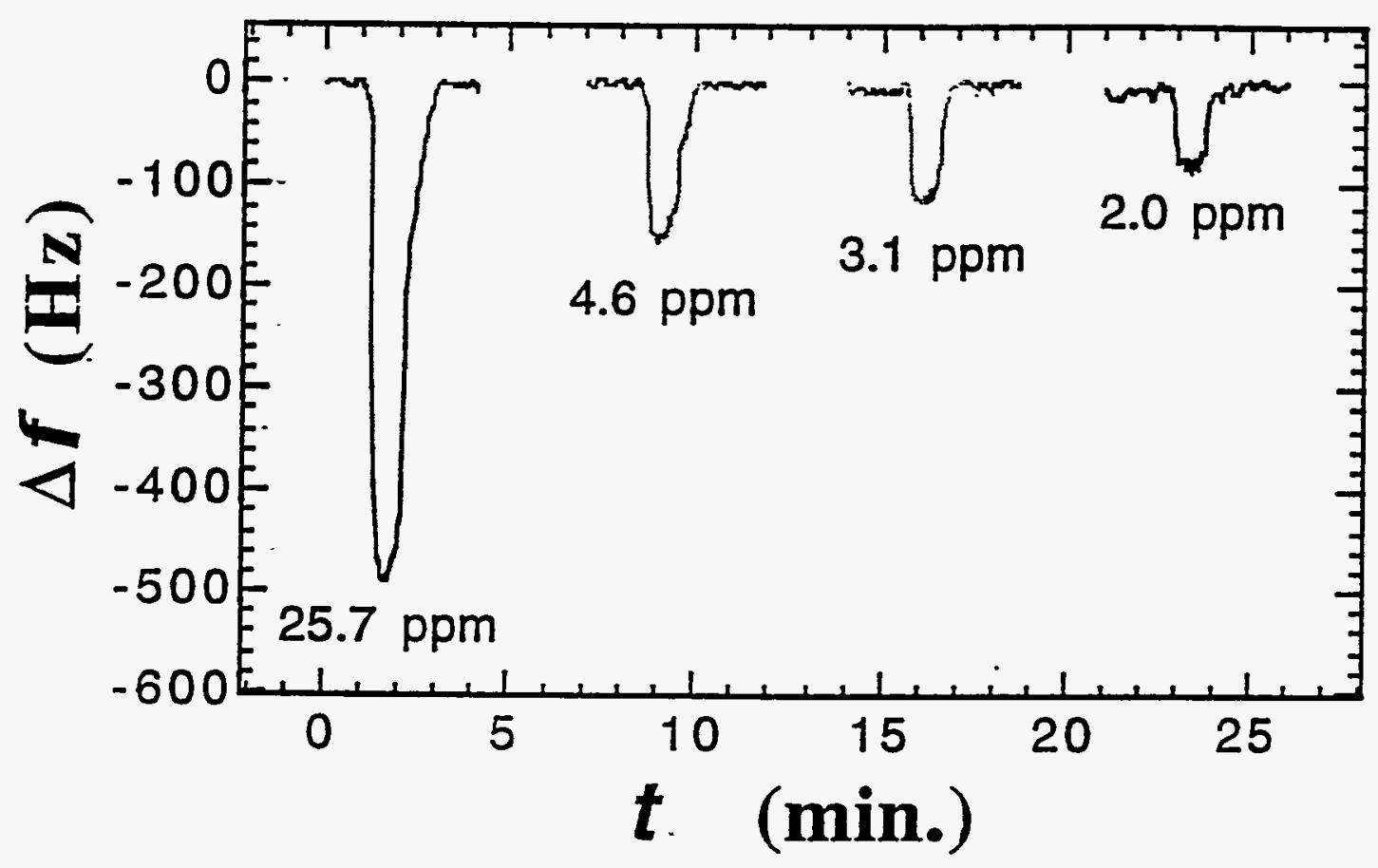

Figure 1. The cyclodextrin-coated, surface-acoustic-wave-based sensor responds to 1-minute-wide acetone pulses at the particular concentrations shown. 